Article

\title{
Development of Mirror Coatings for Gravitational Wave Detectors
}

\author{
Stuart Reid ${ }^{1, *}$ and Iain W. Martin ${ }^{2}$ \\ 1 SUPA, Institute of Thin Films, Sensors \& Imaging, School of Engineering and Computing, \\ University of the West of Scotland, Paisley PA1 2BE, UK \\ 2 SUPA, Institute for Gravitational Research, School of Physics and Astronomy, University of Glasgow, \\ Glasgow G12 8QQ, UK; Iain.Martin@glasgow.ac.uk \\ * Correspondence: Stuart.Reid@uws.ac.uk
}

Academic Editor: Desmond Gibson

Received: 20 August 2016; Accepted: 25 October 2016; Published: 16 November 2016

\begin{abstract}
The first detections of gravitational waves, GW150914 and GW151226, were associated with the coalescence of stellar mass black holes, heralding the opening of an entirely new way to observe the Universe. Many decades of development were invested to achieve the sensitivities required to observe gravitational waves, with peak strains associated with GW150914 at the level of $10^{-21}$. Gravitational wave detectors currently operate as modified Michelson interferometers, where thermal noise associated with the highly reflective mirror coatings sets a critical limit to the sensitivity of current and future instruments. This article presents an overview of the mirror coating development relevant to gravitational wave detection and the prospective for future developments in the field.
\end{abstract}

Keywords: gravitational waves; optical coatings; $1064 \mathrm{~nm}$; ion beam deposition; molecular beam epitaxy

\section{Introduction}

Detecting gravitational waves has been one of the most challenging experimental projects ever to be undertaken. Gravitational waves are predicted from Einstein's general theory of relativity, and are associated with the acceleration of mass [1], with the largest signals expected from violent astronomical events. In September and December 2015, Advanced LIGO (aLIGO) [2] detected gravitational waves associated with the merger of stellar mass black holes [3,4], opening a new area of observational astronomy and shedding new insight on the relativistic nature of our Universe. General relativity describes gravitational waves as fluctuating quadrupolar tidal strains in space, which thus produce differential strains along perpendicular paths in space. This differential strain can be measured by suspending mirrors (to isolate from environmental effects) a distance apart, then using laser interferometry to monitor their relative positions. In the case of Advanced LIGO, the core optical configuration is that of a simple Michelson interferometer, comprising a beamsplitter (BS) and mirrors $\left(\mathrm{M}_{x}\right)$. Fabry-Perot cavities (FP) are incorporated within the $4 \mathrm{~km}$ arms, in addition to power recycling (PR) and signal recycling (SR) mirrors at the input and output ports, respectively. A simple schematic is shown in Figure 1. This paper will specifically discuss the status and future requirements of the coating technology required to make the highly reflective mirrors in these instruments. Further details of how gravitational wave detectors operate, the variety of astrophysical sources that might be observed, and the main sources of fundamental noise expected within these instruments, is detailed elsewhere, for example by Pitkin et al. [5]. 


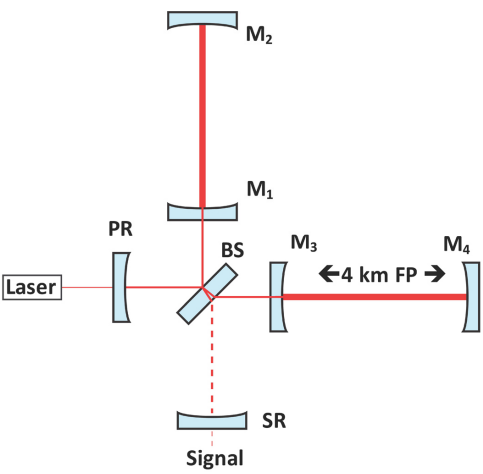

(a)

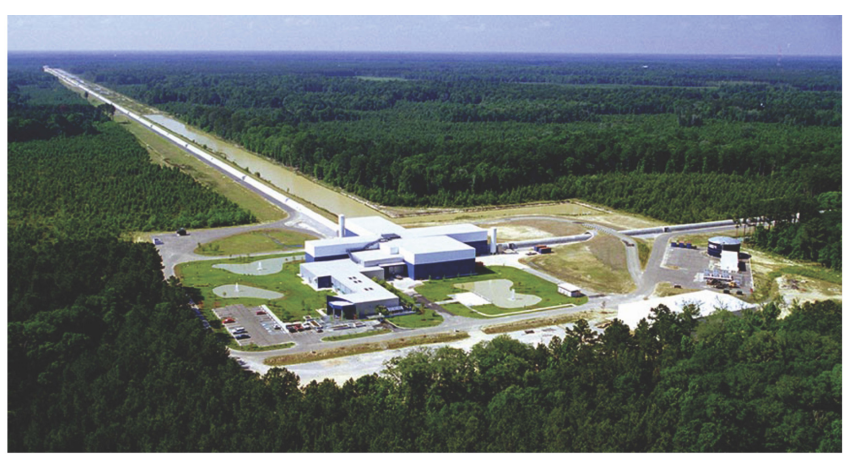

(b)

Figure 1. (a) Simplified schematic interferometric configuration used in Advanced LIGO; (b) LIGO Livingston gravitational wave detector.

To achieve the sensitivities required to detect gravitational waves, significant optical power is required inside the optical cavities of the interferometers in order to reduced shot noise [6]. This sets a formidable challenge on the required mirror specifications, particularly in relation to the optical losses associated with absorption and scatter. Optical losses will limit the achievable optical gain within the cavities. Optical absorption will also heat the mirrors, which can adversely affect interferometer stability (e.g., thermal lensing). Furthermore, optical scatter produces stray light that may reenter the interferometer and create unwanted noise. In the case of Advanced LIGO, the reflectivity required is $>99.9 \%$, with absorption levels of $<0.5 \mathrm{ppm}$, and scatter losses per mirror of $<2 \mathrm{ppm}$ [7]. The only method by which this can be achieved over large areas (diameter $=34 \mathrm{~cm}$ ) is by using multilayer interference coatings fabricated using ion-beam deposition (IBD) [8].

\section{Thermal Noise}

Thermal noise associated with dielectric multilayer coatings sets an important limit in precision measurement applications, such as highly frequency-stabilized lasers [9-11], atomic clocks [12,13], and gravitational wave detectors [14-17]. We discuss here the connections between thermal noise and elastic dissipation in mirrors, and between that dissipation and the structure of amorphous films.

The first reported observation of thermally-driven displacement was by Brown in 1828, of the irregular motion of pollen grains suspended in water [18]. It was not until 1905 that a full mathematical treatment of Brownian motion was presented by Einstein [19]. It is now well understood that any parameter characterizing a dissipative system will exhibit spontaneous thermal fluctuations, as described by the fluctuation-dissipation theorem [20-22]. The frequency-dependent behavior of these fluctuating motions, $S_{x}(f)$, (the power spectral density of the fluctuations) is associated with the dissipative part of the system's impedance, $Z(f)$, and can be shown to be:

$$
S_{x}(f)=\frac{4 k_{\mathrm{B}} T}{4 \pi f^{2} \mathbb{R}\{Z(f)\}}
$$

Where $k_{\mathrm{B}}$ is Boltzmann's constant, $T$ is the temperature, and $f$ is the frequency. The thermal fluctuations (noise) are, therefore, related to the real part of the mechanical impedance, which is the dissipative part and, therefore, represented by the damping coefficient of the system. This can be in the form of external damping, such as gas damping, recoil damping, and / or electrostatic damping, however, external damping effects are intentionally minimized in the design of gravitational wave detectors. Internal damping (friction) arises from anelasticity, where the strain response in a material is not instantaneous when a stress is applied $[23,24]$. The phase lag between the stress and the strain is known as the mechanical loss angle, or the mechanical dissipation factor. In the following section, 
the importance of the mechanical dissipation factor for the thermal noise of high-reflection (HR) coatings will be discussed.

\section{Mechanical Dissipation in Thin Film Coatings}

The mechanical dissipation factor can be defined as:

$$
\phi\left(f_{0}\right)=\frac{E_{\text {lost per cycle }}}{2 \pi E_{\text {total }}} \equiv \frac{\Delta f}{f_{0}}=\frac{1}{Q}
$$

where $E_{\text {total }}$ is the total energy stored in the oscillating system, $E_{\text {lost per cycle }}$ is the energy dissipated per cycle of the oscillation, $\Delta f$ is the width of the resonance peak (FWHM), and $Q$ is the mechanical quality factor.

Within the gravitational wave community, the mechanical dissipation associated with optical coatings has been primarily measured using mechanical ringdown experiments on cylindrical samples, disks, or flexures/cantilevers. In this case, a resonant mode of a sample is excited and then left to freely decay. If the external sources of damping are minimized, then the mechanical dissipation, $\phi\left(f_{0}\right)$, can be calculated from the time-dependent amplitude decay, $A(t)$ :

$$
A(t)=A_{0} \mathrm{e}^{-2 \pi \phi\left(f_{0}\right) f_{0} t / 2}
$$

where $A_{0}$ is the initial excitation amplitude and $f_{0}$ is the resonant frequency. Further details of the experimental techniques can be found in the literature $[25,26]$.

The mechanical loss associated with mirror coatings is typically calculated by measuring the mechanical loss of identical (or nominally identical) substrates, one with a coating and the other uncoated (control). The coating loss is then calculated from the difference between the two samples, with a scaling factor that provides the proportional energy stored in the coating for the distortion associated with the resonant mode [27]. For some coating and substrate combinations, it can also be important to remove the coating thermoelastic loss, due to heat flow associated with the different thermomechanical properties of the coating and substrate $[28,29]$. The coating loss, $\phi\left(f_{0}\right)_{\text {coating }}$ can, therefore, be expressed as [30,31]:

$$
\phi\left(f_{0}\right)_{\text {coating }}=\frac{E_{\mathrm{S}}}{E_{\mathrm{C}}}\left(\phi\left(f_{0}\right)_{\text {coated }}-\phi\left(f_{0}\right)_{\text {substrate }}\right)-\phi\left(f_{0}\right)_{\text {coating thermoelastic }}
$$

where $\phi\left(f_{0}\right)_{\text {coating }}$ is the measured mechanical loss of the coated sample, and $\phi\left(f_{0}\right)_{\text {substrate }}$ is the loss of the uncoated substrate (control), and $E_{S}$ and $E_{C}$ are the elastic strain energies stored in the substrate and coating, respectively.

\section{Coating Thermal Noise Calculation}

Work carried out by Levin [32], and later by Lui and Thorne [33], quantified the importance of the location of the mechanical losses within the interferometer mirrors in a gravitational wave detector. The approach relies on a direct application of the fluctuation-dissipation theorem to the interferometer readout (the probing incident laser beam) on the front face of the mirror. In this case, the thermal noise is calculated by applying a hypothetical pressure on the front face of the mirror, with same intensity distribution as the incident laser beam. The power spectral density of the thermal displacement $S_{x}(f)$ then becomes:

$$
S_{x}(f)=\frac{2 k_{\mathrm{B}} T}{\pi^{2} f^{2}} \frac{W_{\text {diss }}}{F_{0}^{2}}
$$

where $F_{0}$ is the amplitude of the force, and $W_{\text {diss }}$ is the power dissipated due to the application of this force. (Noise equations are typically presented in the form of power spectral density. The amplitude spectral density of the thermal noise, measured in $\mathrm{m} / \sqrt{ }(\mathrm{Hz})$, is given by the square root of this quantity). The value of this dissipation term can be calculated by integrating the force across the 
volume of the mirror, taking into account the elastic properties and mechanical dissipation at each location. In the simple case of homogeneous mechanical loss, and where the incident laser beam is considerably smaller than the mirror radius, the Brownian thermal noise, $S_{x}^{\mathrm{ITM}}(f)$, can be shown to be:

$$
S_{x}^{\mathrm{ITM}}(f)=\frac{2 k_{\mathrm{B}} T}{\sqrt{\pi^{3}} f} \frac{1-\sigma}{Y \omega_{\mathrm{m}}} \phi_{\text {substrate }}(f)
$$

where $\phi_{\text {substrate }}$ is the mechanical dissipation of the substrate material, $Y$ and $\sigma$ are the Young's modulus and Poisson's ratio, and $\omega_{\mathrm{m}}$ is the radius of the incident beam (where electric field amplitude falls to 1 /e of the maximum value). Nakagawa et al. extended these calculations in order to approximate the thermal noise in a coated mirror by assuming the coating to be a thin surface layer of thickness and mechanical loss $\phi_{\text {coating }}[34]$. Applying the Levin approach eventually leads to the expression for the thermal noise of a coated optic to be [35]:

$$
S_{x}(f)=\frac{4 k_{\mathrm{B}} T}{\pi f} \frac{\left(U_{\mathrm{B}} \phi_{\mathrm{B}}+U_{\mathrm{S}} \phi_{\mathrm{S}}\right)}{F_{0}^{2}}
$$

where $U_{\mathrm{B}}$ and $U_{\mathrm{S}}$ are the strain energies associated with bulk and shear displacements, and $\phi_{\mathrm{B}}$ and $\phi_{\mathrm{S}}$ are the mechanical losses of the coating associated with bulk and shear motions. Assuming the mechanical losses associated with bulk and shear motions are equal, $\phi_{\mathrm{B}}=\phi_{\mathrm{S}}=\phi_{\mathrm{C}}\left(\phi_{\mathrm{C}}=\right.$ coating mechanical loss), the thermal noise of the coating can be written as:

$$
s_{x}(f)=\frac{4 k_{\mathrm{B}} T}{\pi^{2} f} \frac{\left(1+\sigma_{\mathrm{S}}\right)\left(1-2 \sigma_{\mathrm{S}}\right)}{E_{\mathrm{S}}} \frac{d}{w_{\mathrm{m}}} \phi_{\mathrm{C}}
$$

This equation highlights a number of key aspects of coating thermal noise. For example, the thermal noise amplitude will depend on the square root of the loss-thickness product, in addition to scaling inversely with the probing laser beam radius. Therefore, strategies for reducing Brownian thermal noise in precision experiments are typically targeted on some combination of (a) reducing the mechanical dissipation associated with mirror coatings, (b) minimizing the required coating thickness, and (c) increasing the laser beam diameter. It should be noted that future experiments also plan to cryogenically cool the suspended mirrors to directly reduce thermal noise through the reduction of the total thermal energy in the mirror [36,37]. However, the mechanical loss of some materials is strongly temperature dependent and, thus, cooling does not always result in a reduction in thermal noise. In particular, the mirror substrates of current detectors are made from fused silica. This material is not suitable for use at low temperature as the mechanical loss increases by approximately five orders of magnitude due to a broad peak centered around $40 \mathrm{~K}$, resulting in a significant increase in thermal noise from cooling to these temperatures. A change in mirror substrate is, therefore, required for cryogenic detectors, with crystalline silicon and sapphire being the most studied candidate materials (discussion of this is detailed in [36]). It should be noted that silicon is not transparent at the current wavelength of $1064 \mathrm{~nm}$ and, thus, to enable light to couple into the arm cavities of the interferometers, a change in mirror substrate would also require a change in the laser wavelength used in detectors, possibly to $1550 \mathrm{~nm}$ or $2000 \mathrm{~nm}$. Suitable coatings for use at longer wavelengths and at cryogenic temperature will be discussed in a later section.

\section{Current Status of Mirror Coatings for Gravitational Wave Detectors}

The initial interferometric gravitational wave detectors used multilayer mirror coatings consisting of ion beam-sputtered amorphous silica $\left(\mathrm{SiO}_{2}\right)$ and tantalum pentoxide $\left(\mathrm{Ta}_{2} \mathrm{O}_{5}\right)$, which were demonstrated to achieve close to 1 ppm level total optical losses (absorption plus scatter) [8]. A standard HR mirror coating design was used, in which materials with high and low refractive indices are deposited in alternative layers, each having an optical thickness of $\lambda / 4$, where $\lambda$ is the wavelength of the laser used in the detector (1064 nm for current room temperature detectors). Figure 2 shows 
the design of the Advanced LIGO mirror suspension stack, in addition to a cross-section image of a standard HR coating stack. The mechanical loss of these coatings was found to be dominated by the high index tantala layers, which exhibit a mechanical loss of $\sim 4 \times 10^{-4}$ compared to a loss of $\sim 5 \times 10^{-5}$ for the silica layers [25]. A reduction in the mechanical loss of these coatings was later achieved through the incorporation of titania within the tantala layers by the Laboratoire des Materiaux Avancés (LMA) in Lyon. Titania was understood to pack densely within the tantalum-oxygen structure, and its high melting point could help stabilize the amorphous structure. Initial experiments demonstrated that a $40 \%$ reduction in coating mechanical loss could be achieved with a titania content of $22.5 \%$ (cation concentration) $[38,39]$. Similar reductions were observed in films deposited by different vendors, however, the initial baseline losses appear to vary between vendors [40].



(a)

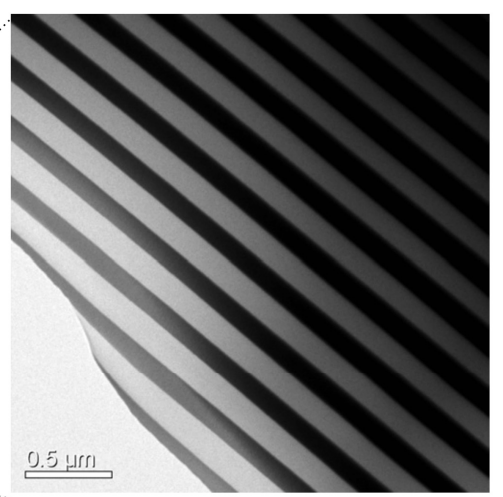

(b)

Figure 2. (a) CAD rendering of the Advanced LIGO mirror suspension; (b) SEM image of an ion beam-sputtered $\lambda / 4$ multilayer stack of $\mathrm{SiO}_{2}$ (light) and $\mathrm{TiO}_{2}$ doped $\mathrm{Ta}_{2} \mathrm{O}_{5}$ (dark) designed for high-reflectivity at $1064 \mathrm{~nm}$. Note the mirror coating is deposited on the front surface of the mirror test mass.

\section{Atomic Structure and Atomic Modeling of Amorphous Coatings}

In order to understand the microscopic processes responsible for mechanical loss in coatings, studies of the atomic structure and composition have been carried out. Fused silica, in both bulk and thin film form, has been extensively characterized in relation to mechanical loss, and is one of the most studied amorphous materials. For bulk samples, a semi-empirical model has been developed [41], where the bulk dissipation is known to arise from the high frequency relaxation in the $\mathrm{Si}-\mathrm{O}$ structure [42]. In the case of gravitational wave detectors, the tantala component of the multi-layer coatings contributes most strongly to the coating thermal noise. Tantala, in thin film, amorphous form, is not a well-understood material. The first attempt within the gravitational wave community to study the origin of the loss mechanism in tantala involved measuring the mechanical loss of tantala coatings across a range of temperatures, since many materials exhibit dissipation peaks with particular temperature and frequency dependences [43]. These dissipation peaks are related to double-well potentials, characterized by an activation energy and a characteristic time constant, and can be associated with discrete mechanical dissipation mechanisms [44]. A dissipation peak was observed at around $20 \mathrm{~K}$ in amorphous tantala coatings, with titania doping reducing the height and increasing the width of the dissipation peak [45]. The activation energy of the dissipation peaks 
observed in tantala are of the same order of magnitude as those observed in fused silica and, therefore, similar-scale atomic transitions may be responsible [46].

Studies of the short- and medium-range order of ion beam-sputtered amorphous tantala coatings have been conducted $[47,48]$ using transmission electron microscopy (TEM) and reduced density function (RDF) analysis, and have shown correlations between the mechanical dissipation and the distribution of atom-atom distances. As the post-heat treatment is increased, the distribution of bond lengths decreases (towards those expected for crystalline $\mathrm{Ta}_{2} \mathrm{O}_{5}$ ), and the room temperature mechanical losses reduce. Figure 3 shows a simulated atomic structure based on the described RDF analysis.



Figure 3. Predicted atomic structure of ion beam-sputtered tantala (dark $=\mathrm{O}$, light $=\mathrm{Ta}$ ).

Computational simulations have been developed using molecular dynamics [49] which are able to predict various features that have been observed in the dissipation of tantala. The most recent simulations support the hypothesis that the medium range structure, associated with atomic ring networks of 10-20 atoms, dominates the mechanical dissipation processes [50]. For this reason, deposition parameters and post-deposition treatment protocols likely need to be optimized in order to further reduce mechanical loss associated with these coating materials.

\section{Routes for Reducing Coating Thermal Noise}

Various potential solutions for reducing the thermal noise associated with mirror coatings within precision optical cavities are currently being explored. It is likely that new discoveries will be made over the coming decade that will revolutionize the understanding of the dissipation mechanisms, and allow the development of techniques to fabricate highly-reflective mirror coatings that satisfy the optical requirements whilst providing a significant reduction in thermal noise. The following subsections will discuss some potential routes for achieving this.

\subsection{Optimisation of Coating Layer Thickness}

In a standard $\mathrm{SiO}_{2} / \mathrm{Ta}_{2} \mathrm{O}_{5}$ mirror coating for $\mathrm{GW}$ detectors, in which each coating layer has an optical thickness of $\lambda / 4$, the total mechanical loss is dominated by the tantala layers. It is possible to reduce the total mechanical loss of the coating by optimizing the thicknesses of the high and low index layers to reduce the total tantala content of the coating, while still maintaining the desired reflectively through the inclusion of additional bi-layers [51]. Optimized coatings have been tested and the theoretical coating thermal noise reduction of $9 \%$ has been experimentally verified through direct thermal noise measurements [52]. The coating optimization procedure can be extended to dichroic coatings, allowing different transmissions at two wavelengths [53], which finds application in, e.g., the aLIGO detectors in enabling the use of a $532 \mathrm{~nm}$ laser for lock acquisition.

\subsection{Optimisation of Deposition Parameters}

It is well-known that ion beam-sputtered silica/tantala coatings from different vendors can exhibit varying levels of mechanical loss. Although not well-understood, this clearly indicates that differences in the deposition parameters can play an important role. Studies on the role of deposition parameters on the optical properties of ion-beam sputtered coatings have been investigated [54,55], however, 
the effect on mechanical loss are minimal to date. The effect of sputtering from xenon gas instead of argon was studied, showing an insignificant effect on the level of mechanical loss [40]. Experiments have also suggested that oxygen deficiency in tantala coatings can increase the associated mechanical loss. The use of ion-assist (or plasma-assist) is well documented for controlling the stress, stability, stoichiometry, and density of both IBD and non-IBD films [56] and currently plays an important role in high performance optical applications, such as those requiring a high laser-damage threshold (LDT) [57]. In relation to mechanical loss, the use of ion assistance is observed to affect the level of mechanical loss in mirror coatings, however, this has not been used to reduce the mechanical loss below single-ion beam deposition [54,58].

The substrate temperature during deposition is an important parameter, which was first reported and characterized by Movchan and Demchishin [59], and then by Thornton [60]; however, these studies used deposition techniques that use lower deposition energies/kinetics than ion-beam deposition. Nevertheless, increasing the deposition temperature has been demonstrated to change the coating structure from a porous, columnar microstructure, towards a dense, polycrystalline structure (referred to as structural zones of condensate). Many deposition procedures use this theory to optimize both density and structure, where $T$ is the substrate/deposition temperature, and $T_{\mathrm{m}}$ is the melting point (or transition temperature) of the coating material:

- $\quad T / T_{\mathrm{m}}<1$ : minimal adatom surface mobility, coating is defined by flux kinetics

- $\quad T / T_{\mathrm{m}}(0.1-0.3)$ : self-diffusion results in growth of dense fibrous grains

- $T / T_{\mathrm{m}}(0.3-0.5)$ : increased surface mobility promotes localized crystallization, columnar growth separated by grain boundaries

- $\quad T / T_{\mathrm{m}} \sim 0.5$ : structures defined by equilibrium conditions, epitaxial growth from crystalline column faces

Although the role of temperature has been important in many deposition processes, we note that ion-beam deposition was originally found to produce highly smooth, amorphous, high-density, films without the use of elevated temperatures, suitable for precision optical applications with very low levels of optical loss (originally for ring laser gyroscopes [61], then exploited within various other high-end applications, such as dense wavelength division multiplexing (DWDM) filters within the telecoms industry). The effect of deposition temperature on the mechanical loss of evaporated silicon films has been shown to be very important, resulting in losses that cannot be achieved through post-deposition heat treatment alone [62]. Of particular note, the role of ion energy on the optical and mechanical properties of optical coatings has not been well-characterized, and comparisons of the effect of ion energy and the effect of substrate temperature during deposition may be of significant interest.

\subsection{Alternative High-Index Amorphous Materials}

Alternative high-index materials to $\mathrm{Ta}_{2} \mathrm{O}_{5}$ have been considered, and the range of candidate materials can be extended if other wavelengths can be used for the laser in future gravitational wave detectors. Currently, detectors operate at a laser wavelength of $1064 \mathrm{~nm}$, which typically limits the candidate high-index materials to oxide materials, such as $\mathrm{ZrO}_{2}, \mathrm{TiO}_{2}$, and $\mathrm{Sc}_{2} \mathrm{O}_{3}$, or perhaps some optimal "alloy" of these materials that enables an atomic structure with minimal mechanical loss. Some promising results for niobia coatings have been reported [40], and even with slightly higher mechanical loss than titania-doped tantala, niobia can potentially achieve similar coating thermal noise to titania-doped tantala due to a more optimal Young's modulus and a relatively high refractive index.

If longer wavelengths can be utilized, non-oxide materials, such as silicon or germanium, could be considered. The mechanical loss of silicon has been reported to reduce by orders of magnitude through elevated temperature deposition [62] and through hydrogenation [63]. It is also important to note that hydrogenation can be used to reduce the IR absorption and shift the absorption edge in sputtered films (associated with the bandgap structure) to lower wavelengths [64] which could be 
beneficial for reducing the optical absorption of silicon and germanium films to a level in which they could be used in future gravitational wave detectors at $1550 \mathrm{~nm}$ or $2 \mu \mathrm{m}$.

Silicon nitride is a potentially useful material for thermal noise reduction, with some films exhibiting mechanical losses of at $1 \times 10^{-6}$ low temperature, more than two orders of magnitude lower than the loss of tantala at these temperatures $[65,66]$ The refractive index of silicon nitride is 2.05 (at $1550 \mathrm{~nm}$ ), leading to the interesting possibility of using it as a low-index component of a coating with aSi $(n=3.4)$ as the high-index material.

\subsection{Alternative Low-Index Amorphous Materials}

For current GW mirror coatings at room temperature, the mechanical loss of the silica layers is not high enough to contribute significantly to the total coating loss. However, the loss of silica films increases at low temperature, with loss peaks observed around 150 and $20 \mathrm{~K}$ [67], close to temperatures proposed for the operation of future GW detectors. At low temperature, the loss of the silica layers will limit the thermal noise performances of coatings using, e.g., aSi, as a high-index layer [68], and of coatings based on a multi-material design (see next section). Reducing the loss of the low-index material is, therefore, also critical for coatings for cryogenic application. This is an area in which more study is likely to be required as, to date, most effort has focused on the high-index materials, which limit coating loss at room temperature. Possible candidate low index materials include alumina, and possibly some fluoride coatings, as they can have a very low refractive index and may, therefore, enable significant reductions in total coating thickness.

\subsection{Multi-Material Coatings}

A reduction in coating thermal noise can be obtained by reducing the total coating thickness, as shown in Equation (8). One method of reducing thickness is to increase the index of the high-index layers, which can reduce the required coating thickness in two ways. Firstly, the physical thickness of each high-index layer required to achieve an optical thickness of $\lambda / 4$ is lower. Secondly, a larger contrast in the refractive index between the low- and high-index components of the coating results in fewer bi-layers being required to achieve the required reflectivity. The reflectivity of a multilayer stack is given by $R=\left(\left(1-n_{\mathrm{G}}\right) /\left(1+n_{\mathrm{G}}\right)\right)^{2}$ where $n_{\mathrm{G}}=n_{\mathrm{S}}\left(n_{\mathrm{H}}^{(2 N+1)} / n_{\mathrm{L}}{ }^{2 N}\right)$ and $n_{\mathrm{S}}, n_{\mathrm{H}}$, and $n_{\mathrm{L}}$ are the refractive indices of the substrate, high-, and low-index materials respectively. As an example, amorphous silicon has a refractive index of 3.4, compared to an index of 2.03 for tantala, at $\lambda=1064 \mathrm{~nm}$. As a result, an amorphous silicon/silica coating only requires seven bi-layers to achieve the same reflectivity as a 17 bi-layer tantala/silica coating, giving a total reduction in thickness of $63 \%$ and a corresponding reduction in thermal noise (assuming identical mechanical loss) of 39\%.

In reality, aSi can have significantly lower mechanical loss than tantala, resulting in even larger thermal noise improvements than estimated above. However, while aSi is highly attractive from a thermal noise perspective, direct replacement of tantala by aSi is unlikely to be possible due to the high optical absorption observed in commercial ion beam-sputtered aSi coatings [69].

The use of 'multi-material coatings' has been proposed to gain the benefits of low mechanical loss materials, such as aSi, without losing the required optical performance $[70,71]$. This allows the materials with higher optical absorption to be buried deeper into the multilayer structure of the coating, where the electric field intensity is significantly lower and, thus, the absorption requirements are significantly relaxed. One such design in which four bi-layers of aSi/silica are used below eight bi-layers of tantala/silica (see Figure 4) could potentially allow a reduction in coating thermal noise of about $20 \%$ at room temperature. Further improvements could be obtained if the absorption of the aSi could be further reduced. 


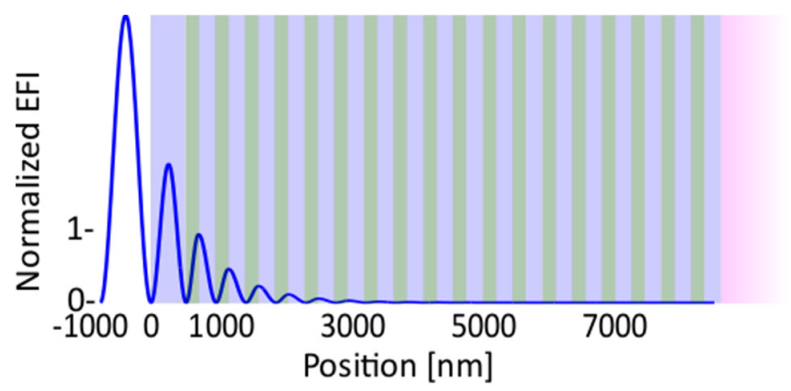

(a)

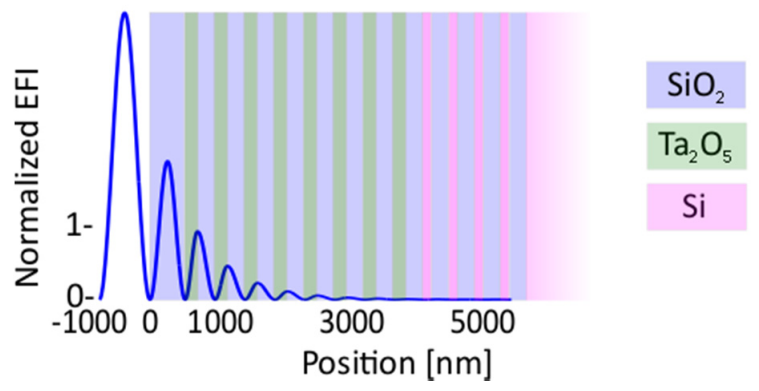

(b)

Figure 4. Illustration of electric field intensity within a standard silica/tantala stack (a) and a mixed material stack (b). In the mixed material model, bilayers of $\mathrm{SiO}_{2}$ and $\mathrm{Ta}_{2} \mathrm{O}_{5}$ are used initially to reduce the laser power, followed by lower layers of amorphous silicon, which can be used to improve thermal noise due to a higher refractive index (reducing thickness and the number of layers) and low mechanical loss.

\subsection{Heat-Treatment and Nano-Layer Coatings}

Post-deposition heat-treatment is known to reduce the mechanical loss of many coating materials, in addition to improving the optical absorption. However, heat-treatment is limited by crystallization of the amorphous films, which can result in higher optical scattering and increased mechanical loss. As noted above, doping can stabilize some amorphous films and increase the crystallization temperature, thus allowing heat-treatment at higher temperatures. For standard silica/tantala coatings, for example, the tantala layers often begin to crystallize when heat-treated at temperatures above $600{ }^{\circ} \mathrm{C}$, while the mechanical loss of the silica layers can be reduced significantly by heat-treatment at temperatures up to $950{ }^{\circ} \mathrm{C}$. Doping tantala with zirconia is known to increase the crystallization temperature [72], and this may be a promising method enabling the use of high temperature heat-treatment to reduce mechanical loss.

Another approach to preventing crystallization is through the use of very thin nano-layers of coating material, in which the small dimensions of the layer suppresses crystallization. Proposals for such nano-layer coatings include the use of a stack of silica/titania nano-layers to form a high-index material. Tests of such coating layers have shown evidence of similar or lower mechanical loss than observed in tantala or titania-doped tantala, with heat-treatment at $300{ }^{\circ} \mathrm{C}$ possible without crystallization of the titania nano-layers [73]. This approach may be useful for exploiting materials which can crystallize easily, such as titania and hafnia, in coatings for use at low temperature, as these materials have already been shown to have low mechanical loss in the cryogenic regime $[74,75]$.

\subsection{Crystalline Mirror Coatings}

Crystalline coatings grown using molecular beam epitaxy (MBE) have been demonstrated to provide a three-fold reduction in Brownian thermal noise [76] within an optical cavity (note that the title of the cited paper refers to a ten-fold reduction in the thermal noise power: here, for consistency 
with conventions in the field, we take the square root of this factor to express the reduction in terms of thermal noise amplitude). These coatings are formed from multilayers of GaAs and AlGaAs, grown on GaAs substrates, and can be transferred onto other substrate materials, e.g., silicon or silica. Excellent optical properties have been demonstrated with these coatings, with total absorption and scatter loss of below 3 ppm obtained between 1064 and $1550 \mathrm{~nm}$, and sub-ppm absorption demonstrated at $1064 \mathrm{~nm}$ [77]. The available diameter of GaAs substrates limits the geometry of these mirrors for use in gravitational wave detectors, with the largest substrates currently available being $200 \mathrm{~mm}$ in diameter. While no evidence of additional thermal noise arising from mechanical loss associated with the coating-substrate bonding procedure was observed in cavity thermal noise measurements [77], further tests to fully evaluate any possible thermal noise associated with larger area bonded coatings may be required.

An alternative technique for utilizing crystalline coatings would be to grow a lattice-matched coating directly onto a suitable crystalline test mass material, removing the need for a complex substrate transfer procedure. In the case of silicon substrates, a potential multilayer crystalline coating solution exists in $\mathrm{GaP} / \mathrm{AlGaP}[78,79]$. Initial tests of a GaP/AlGaP coating have shown significant potential for thermal noise improvements at cryogenic temperatures compared to silica/tantala coatings, with the loss of the GaP/AlGaP being approximately a factor of 60 times lower at $20 \mathrm{~K}$. It should be noted that the $\mathrm{GaP} / \mathrm{AlGaP}$ system is significantly less well studied as a mirror coating than AlGaAs and further development, particularly of the optical properties, is required. Although many MBE processes have been well-developed, the ability to scale-up these coatings to diameters $>34 \mathrm{~cm}$ still requires more research and development. It should be noted that crystalline substrates are not generally suitable for room temperature mirrors due to high levels of thermoelastic noise [80] and, thus, AlGaP coatings are most likely to be of interest for cryogenically-operated detectors.

\subsection{Coating-Free Mirrors}

An alternative solution is to develop a technique where highly-reflective components can be developed without the requirement of deposited thin films. These include diffractively-coupled mirrors [81,82], the use of corner reflectors [83], or the use of waveguide (macro-structured) surfaces [84,85]. These coating-free solutions still require significant development.

\section{Discussion and Conclusions}

With the first detection of gravitational waves from an astrophysical source observed in late 2015, this is an extremely exciting time for gravitational wave astronomy. Future upgrades to the Advanced LIGO detector are planned within the next 2-3 years (e.g., squeezed light states injected to reduce quantum noise), however, a reduction in coating thermal noise is essential to reap the full benefits of future upgrades. In addition, improvements in coating thermal noise will be required for future generations of gravitational wave observatories. Researchers from across the LIGO, Virgo, and KAGRA collaborations are working to address these challenges. Some future detectors will likely operate at different wavelengths and temperatures and, thus, place differing requirements on the mirror coatings. Possible routes to reduced coating thermal noise include improved amorphous oxide coatings; the use of non-oxides, such as amorphous silicon or germanium, perhaps facilitated by multi-material designs to mitigate the effect of optical absorption; and the use of crystalline coatings or the replacement of traditional coated mirrors with diffractive optics. Understanding of dissipation mechanisms in coating materials is increasing through structural measurements and modelling, and the output of this work is beginning to inform research into improved coating materials. The outcome of this research will not just provide a means to search for weaker gravitational wave signals from further out in the Universe, but will also provide underpinning theoretical understanding of how the atomic structure in thin films can affect the macroscopic physical properties of these materials. This will help inform the development of "materials by design", which could benefit a large range of optical and mechanical applications where thin films are utilized. 
Acknowledgments: The authors would like to thank the University of the West of Scotland, the University of Glasgow, the Science and Technology Facilities Council (STFC), the Royal Society, the Royal Society of Edinburgh and the Society of Chemical Industry for financial support. The authors also thank their colleagues within GEO, the LIGO Scientific Collaboration, and Tim Mollart for advice and support, and would like to particularly thank Matthew Abernathy for detailed comments on the manuscript.

Author Contributions: S. Reid and I. W. Martin both contributed equally to the writing of this review paper.

Conflicts of Interest: The authors declare no conflict of interest.

\section{References}

1. Einstein, A. Die Grundlage der allgemeinen Relativitätstheorie. Ann. Phys. 1916, 354, 769-822. (In German) [CrossRef]

2. The LIGO Scientific Collaboration. Advanced LIGO. Class. Quantum Gravity 2015, 32, 074001.

3. Abbott, B.P.; Abbott, R.; Abbott, T.D.; Abernathy, M.R.; Acernese, F.; Ackley, K.; Adams, C.; Adams, T.; Addesso, P.; Adhikari, R.X.; et al. Observation of gravitational waves from a binary black hole merger. Phys. Rev. Lett. 2016, 116, 061102. [CrossRef] [PubMed]

4. Abbott, B.P.; Abbott, R.; Abbott, T.D.; Abernathy, M.R.; Acernese, F.; Ackley, K.; Adams, C.; Adams, T.; Addesso, P.; Adhikari, R.X.; et al. GW151226: Observation of gravitational waves from a 22-solar-mass binary black hole coalescence. Phys. Rev. Lett. 2016, 116, 241103. [CrossRef] [PubMed]

5. Pitkin, M.; Reid, S.; Rowan, S.; Hough, J. Gravitational wave detection by interferometry (ground and space). Living Rev. Relativ. 2011, 14, 13-20. [CrossRef]

6. Edelstein, W.A.; Hough, J.; Pugh, J.R.; Martin, W. Limits to the measurement of displacement in an interferometric gravitational radiation detector. J. Phys. E Sci. Instrum. 1978, 11, 895-897. [CrossRef]

7. Gustafson, E.K.; Shoemaker, D.; Strain, K.; Weiss, R. LSC White Paper on Detector Research and Development, LIGO T990080-00-D. Available online: https://dcc.ligo.org/T990080/public (accessed on 18 August 2016).

8. Ueda, A.; Uehara, N.; Uchisawa, K.; Ueda, K.; Sekiguchi, H.; Mitake, T.; Nakamura, K.; Kitajima, N.; Kataoka, I. Ultra-High Quality Cavity with 1.5 ppm Loss at $1064 \mathrm{~nm}$. Opt. Rev. 1996, 3, 369-372. [CrossRef]

9. Rafac, R.J.; Young, B.C.; Beall, J.A.; Itano, W.M.; Wineland, D.J.; Bergquist, J.C. Sub-dekahertz ultraviolet spectroscopy of ${ }^{199} \mathrm{Hg}+$. Phys. Rev. Lett. 2000, 85, 2462-2465. [CrossRef] [PubMed]

10. Ludlow, A.D.; Huang, X.; Notcutt, M.; Zanon-Willette, T.; Foreman, S.M.; Boyd, M.M.; Blatt, S.; Ye, J. Compact, thermal-noise-limited optical cavity for diode laser stabilization at $1 \times 10^{15}$. Opt. Lett. 2007, 32, 641-643. [CrossRef] [PubMed]

11. Webster, S.A.; Oxborrow, M.; Pugla, S.; Millo, J.; Gill, P. Thermal-noise limited optical cavity. Phys. Rev. A 2008, 77, 033847. [CrossRef]

12. Schmidt-Kaler, F.; Gulde, S.; Riebe, M.; Deuschle, T.; Kreuter, A.; Lancaster, G.; Becher, C.; Eschner, J.; Häffner, H.; Blatt, R. The coherence of qubits based on single $\mathrm{Ca}^{+}$ions. J. Phys. B At. Mol. Opt. Phys. 2003, 36, 623-636. [CrossRef]

13. Miller, R.; Northup, T.E.; Birnbaum, K.M.; Boca, A.; Boozer, A.D.; Kimble, H.J. Trapped atoms in cavity QED: Coupling quantized light and matter. J. Phys. B At. Mol. Opt. Phys. 2005, 38, S551. [CrossRef]

14. Abramovici, A.; Althouse, W.; Camp, J.; Durance, D.; Giaime, J.A.; Gillespie, A.; Kawamura, S.; Kuhnert, A.; Lyons, T.; Raab, F.J.; et al. Improved sensitivity in a gravitational wave interferometer and implications for LIGO. Phys. Lett. A 1996, 218, 157-163. [CrossRef]

15. Lück, H.; The GEO 600 Collaboration. Status of the GEO 600 detector. Class. Quantum Gravity 2006, 23, S71-S78.

16. Acernese, F.; The Virgo Collaboration. The status of VIRGO. Class. Quantum Gravity 2006, 23, S63-S69. [CrossRef]

17. Somiya, K. Detector configuration of KAGRA-The Japanese cryogenic gravitational-wave detector. Class. Quantum Gravity 2012, 29, 124007. [CrossRef]

18. Brown, R. A brief account of microscopical observations made in the months of June, July and August, on the particles contained in the pollen of plants; and on the general existence of active molecules in organic and inorganic bodies. Philos. Mag. 1827, 4, 161-173.

19. Einstein, A. On the movement of small particles suspended in a stationary liquid demanded by the molecular-kinetic theory of heat. Ann. Phys. 1905, 17, 549. [CrossRef] 
20. Callen, H.B.; Welton, T.A. Irreversibility and Generalized Noise. Phys. Rev. 1951, 83, 34-40. [CrossRef]

21. Greene, R.F.; Callen, H.B. On the formalism of thermodynamic fluctuation theory. Phys. Rev. 1951, 83, 1231-1235. [CrossRef]

22. Callen, H.B.; Greene, R.F. On a theorem of irreversible thermodynamics. Phys. Rev. 1952, 86, 702-710. [CrossRef]

23. Zener, C. Elasticity and Anelasticity in Metals; University of Chicago Press: Chicago, IL, USA, 1948.

24. Nowick, A.S.; Berry, B.S. Characterization of Anelastic Behavior. In Anelastic Relaxation in Crystalline Solids; Academic Press: New York, NY, USA, 1972; Chapter 1.

25. Penn, S.D.; Sneddon, P.H.; Armandula, H.; Betzwieser, J.C.; Cagnoli, G.; Camp, J.; Crooks, D.R.M.; Fejer, M.M.; Gretarsson, A.M.; Harry, G.M.; et al. Mechanical loss in tantala/silica dielectric mirror coatings. Class. Quantum Gravity 2003, 20, 2917-2928. [CrossRef]

26. Reid, S.; Cagnoli, G.; Crooks, D.; Hough, J.; Murray, P.; Rowan, S.; Fejer, M.; Route, R.; Zappe, S. Mechanical dissipation in silicon flexures. Phys. Lett. A 2006, 351, 205-211. [CrossRef]

27. Berry, B.S.; Pritchet, W.C. Vibrating reed internal friction apparatus for films and foils. IBM J. Res. Dev. 1975, 19, 334-343. [CrossRef]

28. Fejer, M.M.; Rowan, S.; Cagnoli, G.; Crooks, D.R.M.; Gretarsson, A.; Harry, G.M.; Hough, J.; Penn, S.D.; Sneddon, P.H.; Vyatchanin, S.P. Thermoelastic dissipation in inhomogeneous media: Loss measurements and displacement noise in coated test masses for interferometric gravitational wave detectors. Phys. Rev. D 2004, 70, 082003. [CrossRef]

29. Braginsky, V.B.; Gorodetsky, M.L.; Khalili, F.Y.; Matsko, A.B.; Thorne, K.S.; Vyatchanin, S.P. The noise in gravitational-wave detectors and other classical-force measurements is not influenced by test-mass quantization. Phys. Rev. D 2003, 67, 082001. [CrossRef]

30. Harry, G.M.; Gretarsson, A.M.; Saulson, P.R.; Kittleberger, S.E.; Penn, S.D.; Startin, W.J.; Rowan, S.; Fejer, M.M.; Crooks, D.R.M.; Cagnoli, G.; et al. Thermal noise in interferometric gravitational wave detectors due to dielectric optical coatings. Class. Quantum Gravity 2002, 19, 897-917. [CrossRef]

31. Crooks, D.R.M.; Sneddon, P.; Cagnoli, G.; Hough, J.; Rowan, S.; Fejer, M.M.; Gustafson, E.; Route, R.; Nakagawa, N.; Coyne, D.; et al. Excess mechanical loss associated with dielectric mirror coatings on test masses in interferometric gravitational wave detectors. Class. Quantum Gravity 2002, 19, 883-896. [CrossRef]

32. Levin, Y. Internal thermal noise in the LIGO test masses: A direct approach. Phys. Rev. D 1998, 57, $659-663$. [CrossRef]

33. Liu, Y.T.; Thorne, K.S. Thermoelastic noise and homogeneous thermal noise in finite sized gravitational-wave test masses. Phys. Rev. D 2000, 62, 122002. [CrossRef]

34. Nakagawa, N.; Gretarsson, A.M.; Gustafson, E.K.; Fejer, M.M. Thermal noise in half-infinite mirrors with nonuniform loss: A slab of excess loss in a half-infinite mirror. Phys. Rev. D 2002, 65, 102001. [CrossRef]

35. Hong, T.; Yang, H.; Gustafson, E.K.; Adhikari, R.X.; Chen, Y. Brownian thermal noise in multilayer coated mirrors. Phys. Rev. D 2013, 87, 082001. [CrossRef]

36. ET Conceptual Design Study. Available online: https://tds.ego-gw.it/ql/?c=7954 (accessed on 18 August 2016).

37. LIGO-T1400316-v4: Instrument Science White Paper. Available online: https://dcc.ligo.org/LIGOT1400316/public (accessed on 18 August 2016).

38. Harry, G.M.; Abernathy, M.R.; Becerra-Toledo, A.E.; Armandula, H.; Black, E.; Dooley, K.; Eichenfield, M.; Nwabugwu, C.; Villar, A.; Crooks, D.R.M.; et al. Titania-doped tantala/silica coatings for gravitational-wave detection. Class. Quantum Gravity 2007, 24, 405-415. [CrossRef]

39. Flaminio, R.; Franc, J.; Michel, C.; Morgado, N.; Pinard, L.; Sassolas, B. A study of coating mechanical and optical losses in view of reducing mirror thermal noise in gravitational wave detectors. Class. Quantum Gravity 2010, 27, 084030. [CrossRef]

40. Murray, P. Measurement of the Mechanical Loss of Test Mass Materials for Advanced Gravitational Wave Detectors. Ph.D. Thesis, University of Glasgow, Glasgow, UK, 2008.

41. Penn, S.D.; Ageev, A.; Busby, D.; Harry, G.M.; Gretarsson, A.M.; Numata, K.; Willems, P. Frequency and surface dependence of the mechanical loss in fused silica. Phys. Lett. A 2006, 352, 3-6. [CrossRef]

42. Weidersich, J.; Adichtchev, S.V.; Rossler, E. Spectral Shape of Relaxations in Silica Glass. Phys. Rev. Lett. 2000, 84, 2718. [CrossRef] [PubMed] 
43. Martin, I.; Armandula, H.; Comtet, C.; Fejer, M.M.; Gretarsson, A.; Harry, G.; Hough, J.; Mackowski, J.-M.M.; MacLaren, I.; Michel, C.; et al. Measurements of a low-temperature mechanical dissipation peak in a single layer of $\mathrm{Ta}_{2} \mathrm{O}_{5}$ doped with $\mathrm{TiO}_{2}$. Class. Quantum Gravity 2008, 25, 055005. [CrossRef]

44. Nowick, A.S.; Berry, B.S. Mechanical Models and Discrete Spectra. In Anelastic Relaxation in Crystalline Solids; Academic Press: New York, NY, USA, 1972; Chapter 3.

45. Martin, I.W.; Chalkley, E.; Nawrodt, R.; Armandula, H.; Bassiri, R.; Comtet, C.; Fejer, M.M.; Gretarsson, A.; Harry, G.; Heinert, D.; et al. Comparison of the temperature dependence of the mechanical dissipation in thin films of $\mathrm{Ta}_{2} \mathrm{O}_{5}$ and $\mathrm{Ta}_{2} \mathrm{O}_{5}$ doped with $\mathrm{TiO}_{2}$. Class. Quantum Gravity 2009, 26, 155012. [CrossRef]

46. Fine, M.E.; van Duyne, H.; Kenney, N.T. Low-temperature internal friction and elasticity effects in vitreous silica. J. Appl. Phys. 1954, 25, 402-405. [CrossRef]

47. Bassiri, R.; Borisenko, K.B.; Cockayne, D.J.H.; Hough, J.; MacLaren, I.; Rowan, S. Probing the atomic structure of amorphous $\mathrm{Ta}_{2} \mathrm{O}_{5}$ coatings. Appl. Phys. Lett. 2011, 98, 031904. [CrossRef]

48. Bassiri, R.; Borisenko, K.B.; Cockayne, D.J.H.; Hough, J.; MacLaren, I.; Rowan, S. Probing the atomic structure of amorphous $\mathrm{Ta}_{2} \mathrm{O}_{5}$ mirror coatings for advanced gravitational wave detectors using transmission electron microscopy. J. Phys. Conf. Ser. 2010, 241, 012070. [CrossRef]

49. Hamdan, R.; Trinastic, J.P.; Cheng, H.P. Molecular dynamics study of the mechanical loss in amorphous pure and doped silica. J. Chem. Phys. 2014, 141, 054501. [CrossRef] [PubMed]

50. Trinastic, J.P.; Hamdan, R.; Billman, C.; Cheng, H.-P. Molecular dynamics modeling of mechanical loss in amorphous tantala and titania-doped tantala. Phys. Rev. B 2016, 93, 014105. [CrossRef]

51. Agresti, J.; Castaldi, G.; DeSalvo, R.; Galdi, V.; Pierro, V.; Pinto, I.M. Optimized multilayer dielectric mirror coatings for gravitational wave interferometers. Proc. SPIE 2016, 6286, 628608.

52. Villar, A.E.; Black, E.D.; Desalvo, R.; Libbrecht, K.G.; Michel, C.; Morgado, N.; Pinard, L.; Pinto, I.M.; Pierro, V.; Galdi, V.; et al. Measurement of thermal noise in multilayer coatings with optimized layer thickness. Phys. Rev. D 2010, 81, 122001. [CrossRef]

53. Principe, M.; DeSalvo, R.; Pinto, I.; Galdi, V. Minimum Brownian Noise Dichroic Dielectric Mirror Coatings for AdLIGO. LIGOT080337 2008. Available online: https:/ /dcc.ligo.org (accessed 18 August 2016).

54. Netterfield, R.P.; Gross, M.; Baynes, F.N.; Green, K.L.; Harry, G.M.; Armandula, H.; Rowan, S.; Hough, J.; Crooks, D.R.M.; Fejer, M.M.; et al. Low Mechanical Loss Coatings for LIGO Optics: Progress Report. In Advances in Thin-Film Coatings for Optical Applications II; Proceedings of SPIE; Fulton, M.L., Kruschwitz, J.D.T., Eds.; SPIE: Bellingham, WA, USA, 2005; Volume 5870, p. 58700.

55. Pinard, L.; The Virgo Collaboration. Low Loss Coatings for the VIRGO Large Mirrors. In Advances in Optical Thin Films; Proceedings of SPIE; Amra, C., Kaiser, N., Macleod, H.A., Eds.; SPIE: Bellingham, WA, USA, 2004; Volume 5250, pp. 483-492.

56. Green, J.E.; Barnett, S.A.; Sundgren, J.-E.; Rockett, A. Low-energy ion/surface interaction during film growth from the vapor phase. In Ion Beam Assisted Film Growth; Itoh, T., Ed.; Elsevier: Amsterdam, The Netherlands; New York, NY, USA, 1989.

57. Oliver, J.B.; Kupinski, P.; Rigatti, A.L.; Schmid, A.W.; Lambropoulos, J.C.; Papernov, S.; Kozlov, A.; Spaulding, J.; Sadowski, D.; Chrzan, Z.R.; et al. Large-aperture plasma-assisted deposition of inertial confinement fusion laser coatings. Appl. Opt. 2011, 50, 19-26. [CrossRef] [PubMed]

58. Harry, G. Optical Coatings for Gravitational Wave Detection. LIGO-G040434-00-R 2004. Available online: https:/ / dcc.ligo.org (accessed 18 August 2016).

59. Movchan, B.A.; Demchishin, A.V. Study of Structure and Properties of Bulk Vacuum Condensates of Nickel, Tungsten, Aluminum Oxide, and Zirconium Dioxide. Fiz. Met. Metalloved. 1969, 28, 653.

60. Thornton, J.A. Influence of apparatus geometry and deposition conditions on the structure and topography of thick sputtered coatings. J. Vac. Sci. Technol. 1974, 11, 666-670. [CrossRef]

61. Wei, D.T.; Louderback, A.W. Method for Fabricating Multi-Layer Optical Films. U.S. Patent 4,142,958, 6 March 1979.

62. Liu, X.; Queen, D.R.; Metcalf, T.H.; Karel, J.E.; Hellman, F. Hydrogen-Free Amorphous Silicon with no Tunneling States. Phys. Rev. Lett. 2014, 113, 025503. [CrossRef] [PubMed]

63. Liu, X.; Pohl, R.O. Low-energy excitations in amorphous films of silicon and germanium. Phys. Rev. B 1998, 58, 9067-9081. [CrossRef] 
64. Gibson, D.; Song, S.; Li, C.; Child, D. Optical Properties of Sputter Deposited Amorphous Hydrogenated Silicon Films. In Proceedings of Optical Interference Coatings, Tucson, AZ, USA, 19-24 June 2016; OSA (Optical Society of America): Washington, DC, USA, 2016.

65. Southworth, D.R.; Barton, R.A.; Verbridge, S.S.; Ilic, B.; Fefferman, A.D.; Craighead, H.G.; Parpia, J.M. Stress and Silicon Nitride: A Crack in the Universal Dissipation of Glasses. Phys. Rev. Lett. 2009, 102, 225503. [CrossRef] [PubMed]

66. Chao, S.; Pan, H.-W.; Kuo, L.-C.; Huang, S.-Y.; Wu, M.-Y.; Juang, Y.-H.; Lee, C.-W. Silicon-nitride Films Deposited by PECVD Method on Silicon Substrate for Next Generation Laser Interference Gravitational Wave Detector. In Proceedings of Optical Interference Coatings, Tucson, AZ, USA, 19-24 June 2016; OSA (Optical Society of America): Washington, DC, USA, 2016.

67. Martin, I.W.; Nawrodt, R.; Craig, K.; Schwarz, C.; Bassiri, R.; Harry, G.; Hough, J.; Penn, S.; Reid, S.; Robie, R.; et al. Low temperature mechanical dissipation of an ion-beam sputtered silica film. Class. Quantum Gravity 2014, 31, 035019. [CrossRef]

68. Murray, P.G.; Martin, I.W.; Craig, K.; Hough, J.; Robie, R.; Rowan, S.; Abernathy, M.R.; Pershing, T.; Penn, S. Ion-beam sputtered amorphous silicon films for cryogenic precision measurement systems, Phys. Rev. D 2015, 92, 062001. [CrossRef]

69. Steinlechner, J.; Martin, I.W.; Bassiri, R.; Bell, A.; Fejer, M.M.; Hough, J. Markosyan, A.; Route, R.K.; Rowan, S. Optical Absorption of Ion-Beam Sputtered aSi Coatings. Phys. Rev. D 2016, 93, 062005. [CrossRef]

70. Steinlechner, J.; Martin, I.W.; Hough, J.; Krüger, C.; Rowan, S.; Schnabel, R. Thermal noise reduction and absorption optimization via multimaterial coatings. Phys. Rev. D 2015, 91, 042001. [CrossRef]

71. Yam, W.; Gras, S.; Evans, M. Multimaterial coatings with reduced thermal noise. Phys. Rev. D 2015, 91, 042002. [CrossRef]

72. Tewg, J.-Y.; Kuo, Y.; Jiang, L. Suppression of Crystallization of Tantalum Oxide Thin Film by Doping with Zirconium. Electrochem. Solid-State Lett. 2005, 8, G27-G29. [CrossRef]

73. Pan, H.-W.; Wang, S.-J.; Kuo, L.-C.; Chao, S.; Principe, M.; Pinto, I.M.; DeSalvo, R. Thickness-dependent crystallization on thermal anneal for titania/silica nm-layer composites deposited by ion beam sputter method. Opt. Express 2014, 24, 29847-29854. [CrossRef] [PubMed]

74. Abernathy, M.; Reid, S.; Chalkley, E.; Bassiri, R.; Martin, I.W.; Evans, K.; Fejer, M.M.; Gretarsson, A.; Harry, G.M.; Hough, J.; et al. Cryogenic mechanical loss measurements of heat-treated hafnium dioxide. Class. Quantum Gravity 2011, 28, 195017. [CrossRef]

75. Scott, W.W.; MacCrone, R.K. Apparatus for mechanical loss measurements in low loss materials at audio frequencies and low temperatures. Rev. Sci. Instrum. 1968, 39, 821-823. [CrossRef]

76. Cole, G.D.; Zhang, W.; Martin, M.J.; Ye, J.; Aspelmeyer, M. Tenfold reduction of Brownian noise in high-reflectivity optical coatings. Nat. Photonics 2013, 7, 644-650. [CrossRef]

77. Cole, G.D.; Zhang, W.; Bjork, B.J.; Follman, D.; Heu, P.; Deutsch, C.; Sonderhouse, L.; Robinson, J.; Franz, C.; Alexandrovski, A.; et al. High-performance near- and mid-infrared crystalline coatings. Optica 2016, 3, 647-656. [CrossRef]

78. Lin, A.C.; Bassiri, R.; Omar, S.; Markosyan, A.S.; Lantz, B.; Route, R.; Byer, R.L.; Harris, J.S.; Fejer, M.M. Epitaxial growth of $\mathrm{GaP} / \mathrm{AlGaP}$ mirrors on $\mathrm{Si}$ for low thermal noise optical coatings. Opt. Mater. Express 2015, 5, 1890-1897. [CrossRef]

79. Cumming, A.V.; Craig, K.; Martin, I.W.; Bassiri, R.; Cunningham, L.; Fejer, M.M.; Harris, J.S.; Haughian, K.; Heinert, D.; Lantz, B.; et al. Measurement of the mechanical loss of prototype GaP/AlGaP crystalline coatings for future gravitational wave detectors. Class. Quantum Gravity 2015, 32, 035002. [CrossRef]

80. Braginsky, V.; Gorodetsky, M.; Vyatchanin, S. Thermodynamical fluctuations and photo-thermal shot noise in gravitational wave antennae. Phys. Lett. A 1999, 264, 1-10. [CrossRef]

81. Sun, K.-X.; Byer, R.L. All-reflective Michelson, Sagnac, and Fabry-Perot interferometers based on grating beam splitters. Opt. Lett. 1998, 23, 567-569. [CrossRef] [PubMed]

82. Burmeister, O.; Britzger, M.; Thüring, A.; Friedrich, D.; Brückner, F.; Danzmann, K.; Schnabel, R. All-reflective coupling of two optical cavities with 3-port diffraction gratings. Opt. Express 2010, 18, 9119-9132. [CrossRef] [PubMed]

83. Braginsky, V.B.; Vyatchanin, S.P. Corner reflectors and quantum-nondemolition measurements in gravitational wave antennae. Phys. Lett. A 2004, 324, 345-360. [CrossRef] 
84. Brückner, F.; Clausnitzer, T.; Burmeister, O.; Friedrich, D.; Kley, E.-B.; Danzmann, K.; Tünnermann, A.; Schnabel, R. Monolithic dielectric surfaces as new low-loss light-matter interfaces. Opt. Lett. 2008, 33, 264-266. [CrossRef] [PubMed]

85. Brückner, F.; Friedrich, D.; Clausnitzer, T.; Britzger, M.; Burmeister, O.; Danzmann, K.; Kley, E.-B.; Tünnermann, A.; Schnabel, R. Realization of a monolithic high reflectivity cavity mirror from a single silicon crystal. Phys. Rev. Lett. 2010, 104, 163903. [CrossRef] [PubMed]

(C) 2016 by the authors; licensee MDPI, Basel, Switzerland. This article is an open access article distributed under the terms and conditions of the Creative Commons Attribution (CC-BY) license (http://creativecommons.org/licenses/by/4.0/). 